ago for sick sinus syndrome in other hospital. On physical exam: His Blood pressure was $150 / 80 \mathrm{~mm} \mathrm{Hg}$ and heart rate $55 \mathrm{bpm}$. His lungs were clear on auscultation. Neurological exam was normal. The coronary angiography showed $75 \%$ stenosis in mid segment of right coronary artery (RCA), $50 \%$ in mid segment of anterior descending artery, $60 \%$ from ostium to proximal segment and subtotal occlusion of distal part of circumflex artery. A 3.0*24 mm drug-eluting stent was implanted in RCA and a $2.5^{*} 29 \mathrm{~mm}$ in circumflex artery after pre-dilation. $6 \mathrm{~h}$ later the patient complained pain in xiphoid process, back and neck. The monitor displayed blood pressure $69 / 57 \mathrm{~mm} \mathrm{Hg}$ and heart rate $54 \mathrm{bpm}$. Dopamine was administered with simultaneous transfusion of $250 \mathrm{ml}$ saline and the blood pressure returned to and maintained at $100 / 60 \mathrm{~mm} \mathrm{Hg}$ within $30 \mathrm{~min} .4 \mathrm{~h}$ later, cardiac arrest occurred and the patient lost consciousness. Cardiopulmonary resuscitation was performed immediately and bedside echocardiography found cardiac tamponade. Pericardiaocentesis was performed and $200 \mathrm{ml}$ bloody fluid was withdrawn. Heart beat recovered and blood pressure returned to normal level. $10 \mathrm{~h}$ later, the patient woke up and was talkative, but could not move legs. He also had bladder and rectal incontinence. Neurological evaluation was as follows: cranial nerves without changes, absence of pain from umbilicus down, preserved deep sensitivity, deep tendon reflexes abolished and muscle tone decreased in legs. Computer tomography showed lacunar infarction of brain and degeneration of thoracic spinal column 5-9. Cerebrospinal fluid was clear with total proteins $230.6 \mathrm{mg} / \mathrm{dl}$, WBC 7.0*106/1 and IgG $580.0 \mathrm{mg} / \mathrm{l}$. Anterior spinal artery syndrome was diagnosed and steroid, anti-platelet and anti-coagulation agents, vitamin B and butylphthalide were used. Rehabilitation therapy was introduced one month later. 3 months later, he regained urinary and fecal continence and could stand with a walker. The patient discharged half year later.

Conclusion In older patients with diffuse arteriosclerosis, delayed cardiac tamponade may occur after PCI and induce persistent hypotension, even cardiac tamponade, and result in ASAS. Therefore, close observation and immediate management are very important.

\section{e0340 ACTIONS OF IRBESARTAN ON ATPASE ACTIVITY AND ANGIOTENSIN II IN BLOOD VESSELS FROM RENAL HYPERTENSIVE RATS}

doi:10.1136/hrt.2010.208967.340

${ }^{1}$ Qian-Hui Shang, ${ }^{1}$ Ze-Bing Wu, ${ }^{1}$ Qian-Feng Jiang, ${ }^{2}$ Oin Wu. ${ }^{1}$ Institute of Clinical Medicine of Zunyi Medical College, Department of Cardiology, Affiliated Hospital of Zunyi Medical College; ${ }^{2}$ Department of Pharmacology, Zunyi Medical College, Zunyi Guizhou, China

Objective To explore the effects of irbesartan on activities of $\mathrm{Na}^{+}$$\mathrm{K}^{+}$-ATPase, Ca2 ${ }^{+}$-ATPase, Angiotensin II (AngII) and vascular remodelling in renal hypertensive rats (RHRs).

Methods Renovascular hypertension was induced by two kidneyone clip method. Eighteen RHRs were randomly divided into 3 groups: RHR model group ( $\mathrm{n}=6)$, irbesartan treated group [50 mg/ $(\mathrm{kg} \mathrm{d}), n=6]$, withdrawal group $(n=6)$. Six rats were included in sham operation group. Blood pressure was measured before and after using irbesartan. Thicknesses of vascular wall (TVW) of thoracic aorta and mesenteric artery were measured after 8 weeks. ATPase activities were determined by enzymatic colorimetric method. AngII level was detected by radioimmunoassay.

Results Compared to the sham operation group, blood pressure, TVW, AngII levels of plasma and blood vessels were increased in RHR. The activities of $\mathrm{Na}^{+}-\mathrm{K}^{+}$-ATPase and $\mathrm{Ca} 2^{+}$-ATPase were decreased in RHR. Blood pressure and the TVW of mesenteric artery were significantly decreased by irbesartan treatment. An increased AngII level and activity of $\mathrm{Ca}^{+}$-ATPase in thoracic aorta and mesenteric artery were also found [thoracic aorta: $(11.9 \pm 1.9)$ vs $(7.5 \pm 1.6) \mu \mathrm{mol} \mathrm{Pi} /(\mathrm{h} \cdot \mathrm{mg}$ pro); mesenteric artery: $(11.6 \pm 1.9)$ vs $(8.2 \pm 0.8) \mu \mathrm{mol} \mathrm{Pi} /(\mathrm{h} \cdot \mathrm{mg}$ pro), both $\mathrm{p}<0.01]$. No change of $\mathrm{Na}^{+}-\mathrm{K}^{+}$. ATPase activity was found after irbesartan treatment. After one-week discontinuation of treatment, blood pressure was significantly elevated, the activity of $\mathrm{Ca}^{2+}$-ATPase of thoracic aorta $[(7.6 \pm 1.4) \mu \mathrm{mol} \mathrm{Pi} /(\mathrm{h} \cdot \mathrm{mg}$ pro $)]$ and mesenteric artery $[(6.9 \pm 1.3) \mu \mathrm{mol}$ $\mathrm{Pi} /(\mathrm{h} \cdot \mathrm{mg}$ pro)] was decreased (both $\mathrm{p}<0.01)$. There was a significant negative correlation between AngII and the activity of $\mathrm{Ca}^{2+}$-ATPase in RHR.

Conclusions The vascular remodelling of RHR may be associated with decreased vascular ATPases activities. Irbesartan can reverse vascular remodelling partially by increasing $\mathrm{Ca}^{2+}$-ATPase activity.

\section{e0341 EFFECT OF FASTING GLUCOSE LEVELS ON MORTALITY RATE IN PATIENTS WITH DIABETES MELLITUS AND CORONARY ARTERY DISEASE UNDERGOING REVASCULARIZATION}

doi:10.1136/hrt.2010.208967.341

Kang Junping, Liu Xiaohui, Lv Oiang, Hu Rong, Wu Jiahui, Ma Changsheng. Beijing Anzhen Hospital

Objectives We examined the association between glycaemic control determined by fasting glucose levels before elective PCI and the outcomes in diabetic patients undergoing elective revascularization. Background Patients with diabetes mellitus (DM) have a worse clinical outcome after PCI than patients without DM, but whether optimal glycaemic control before PCI could improve the prognosis is not clear.

Methods The DESIRE-2 (Drug-Eluting Stent Impact on Revascularization-2) was a single-center registry of coronary revascularization in our institution between July 1st 2003 and Sep 30th 2005. A total of 434 diabetic patients undergoing elective PCI were enrolled in this study. Optimal glycaemic control was defined as fasting glucose $<126 \mathrm{mg} / \mathrm{dl}$, and suboptimal control was defined as fasting glucose $>=126 \mathrm{mg} / \mathrm{dl}$. Median follow-up duration after the index intervention was 523 days.

Results The average patient age was $61.0 \pm 9.8$ years; $69.8 \%$ of the patients were men. The patients with optimal glycaemic control were older than the suboptimal control group (62.1 \pm 9.46 vs 59.6 \pm 10.41$)$. Compared with diabetic patients with optimal glycaemic control, those with suboptimal glycaemic control had similar rates of total mortality $(3.3 \%$ vs $3.9 \%, p=0.762)$ and major adverse cardiac and cerebral events $(15.9 \%$ vs $12.4 \%, p=0.308)$. In a multiple Cox regression analysis, total cholesterol level (HR 1.009, 95\% CI 1.002 to $1.016, p=0.013$ ) and number of lesion (HR 2.070, $95 \%$ CI 1.340 to $3.199, p=0.001$ ) were significant independent predictors of MACCE.

Conclusions In diabetic patients undergoing elective PCI, optimal glycaemic control did not improve clinical prognosis. These data suggest that aggressive treatment of DM to achieve fasting glucose $<126 \mathrm{mg} /$ dl before PCI is not necessary.

\section{E0342 EFFECT OF ACARBOSE ON MYOCARDIAL PERFUSION IN PATIENTS WITH CORONARY HEART DISEASE AND IMPAIRED GLUCOSE TOLERANCE AFTER PCI: A CLINICAL TRIAL}

doi:10.1136/hrt.2010.208967.342

Keng Wu, Hongmei Zhao. of Guangdong Medical College

Objective To study the effect of Acarbose on myocardial perfusion in revascularized patients with coronary heart disease and impaired glucose tolerance after percutaneous coronary intervention (PCI) 
and investigate variations of lipid, glucose, and vascular endothelial growth factor (VEGF) levels myocardial perfusion images and cardiac function, as well as the possible mechanisms to improve myocardial perfusion.

Methods Total 102 cases were selected, 5 patients were lost. Finally 97 patients included, 77 males and 20 females, aged 56 \pm 13 . Of whom, 30 cases suffered from acute myocardial infarction (AMI), 48 from acute coronary syndromes (ACS), and 19 from chronic stable angina pectoris. They were randomised into three groups, $A(n=33)$, $B(n=31)$, and $C(n=33)$ without differences in the baseline level. Normal adults were control group $\mathrm{D}(\mathrm{n}=30)$. Groups $\mathrm{A}$ and $\mathrm{B}$ were given by Acarbose $50 \mathrm{mg}$ tid and $100 \mathrm{mg}$ tid, respectively, Groups C and $\mathrm{D}$ were given by placebo, the treatment course lasted three months, and CHD patients of each group underwent the same basic treatments after PCI. Myocardial perfusion imaging and variations of blood lipid, IGT, VEGF levels, myocardial perfusion images and cardiac function were observed.

Results 1 . The IGT had no statistically differences before treatments in A, B and C groups ( $p>0.05$ ). The changes of FPG and OGTT $2 \mathrm{~h}$ PG levels had statistically differences after treatments in $A$ and $B$ groups $(p<0.05)$, especially $B$ group $(p<0.01)$; The changes of HbA1c had no statistically significant differences after treatments in the four groups ( $p>0.05)$; The changes of lipid levels had no statistically differences $(p>0.05)$ before and after treatments in A, B, C and $\mathrm{D}$ groups, respectively. 2. A, B, C groups had the significantly higher plasma VEGF levels $(203 \pm 89 \mathrm{ng} / \mathrm{l}$ vs $77 \pm 52 \mathrm{ng} / \mathrm{l}, \mathrm{p}<0.01$ ) than $\mathrm{D}$ group before treatments. The changes of VEGF levels had statistically differences after treatments in $A$ and $B$ groups $(p<0.05)$, especially B group $(p<0.01)$. The changes of VEGF levels had no statistically differences before and after treatments in $C$ and $D$ groups $(p>0.05)$. 3. The myocardial perfusion images and cardiac function had no statistically differences before treatments in A, B and $C$ groups ( $p>0.05$ ), The changes of myocardial perfusion images had statistically differences after treatments in A and B groups $(p<0.05)$, especially B group $(p<0.01)$; The changes of cardiac function had statistically differences after treatments in $A$ and $B$ groups $(p<0.05)$, there is no statistically differences after treatments between $A$ and $B(p>0.05)$; The changes of myocardial perfusion images and cardiac function had no statistically differences in $\mathrm{C}$ group ( $p>0.05)$.

Conclusions Acarbose can regulates IGT, improve myocardial perfusion images and cardiac function. The mechanisms may include reducing VEGF levels, suppressing endothelial hyperplasia, and improving the microcirculation.

\section{e0343 TRIPLE VERSUS DUAL ANTIPLATELET THERAPY IN PATIENTS WITH ACUTE CORONARY SYNDROME UNDERGOING PERCUTANEOUS CORONARY INTERVENTION}

doi:10.1136/hrt.2010.208967.343

Bin Hu, Yujie Zhou, Yuyang Liu, Dongmei Shi, Yingxin Zhao, Dean Jia. Beijing Anzhen Hospital

Background Following percutaneous coronary intervention (PCI), clopidogrel in addition to aspirin therapy leads to greater protection from thrombotic complications than aspirin alone. Whether triple antiplatelet therapy is superior or similar to dual antiplatelet therapy in patients with acute coronary syndrome undergoing PCI in the era of drug-eluting stents remains unclear.

Objectives To evaluate the effect of triple antiplatelet vs dual antiplatelet therapy in patients with acute coronary syndrome after PCI.

Methods and Results We collected consecutive 1203 acute coronary syndrome patients undergoing drug-eluting stents implantation. They received either dual (aspirin plus clopidogrel; dual group; $\mathrm{n}=682$ ) or triple (aspirin plus clopidogrel plus cilostazol; triple group; $n=521$ ) antiplatelet therapy. The triple group received additional cilostazol at least for 1 month. Various major adverse cardiac events at 1 year were compared between these 2 groups. Compared with the dual group, the triple group had a similar incidence of major bleeding events but a significantly lower incidence of inhospital mortality. Clinical outcomes at 1 year showed that the triple group had significantly lower incidences of cardiac death and total major adverse cardiac events than the dual group.

Conclusions Triple antiplatelet therapy seems to be superior to dual antiplatelet therapy in patients ACS undergoing PCI with drugeluting stents.

\section{e0344 THE MECHANISM RESEARCH OF FRP INHIBITS ENDOTHELIAL CELL APOPTOSIS}

doi:10.1136/hrt.2010.208967.344

Shen Hua, Zhou Yujie, Liu Yuyang, Yang Shiwei, Gao Fei, Wang Zhijian, Shi Dongmei, Han Hongya, Ge Hailong, Liu Xiaoli. Department of Cardiology Beijing Anzhen Hospital Capital Medical University Beijing, China

Background Atherosclerosis is the most common cause of cardiovascular diseases in the world. Although the development of atherosclerosis appears to be the result of multiple maladaptive pathways, a particularly important factor in the pathogenesis of atherosclerosis is oxidised low density lipoprotein (ox-LDL), which contributes to endothelial damage. Data from our lab and others show that Follistatin related protein (FRP), which is expressed in the vasculature, has cardioprotective effects, suggesting that loss of FRP protection might play a role in the development of atherosclerosis. Objective In the present study, we determined whether FRP overexpression protects against endothelial cell (EC) damage, an intermediate endpoint for atherosclerosis.

Methods We bred ApoE knockout (ApoE (-/-)) mice that were FRP+ transgenic (they overexpressed FRP. We compared them to control mice (their littermates). Human umbilical vein endothelial cells (HUVECs) were isolated and treated with ox-LDL and recombinant FRP. FRP-induced signal transduction and $\mathrm{Bc} 2 \mathrm{mRNA}$ and protein stability were analysed.

Results After 16 weeks, ApoE (-/-) FRP (+) mice had significantly fewer apoptotic endothelial cells than controls. In vitro experiments showed that the effect of FRP on EC apoptosis was mediated by upregulation of expression of the antiapoptotic protein $\mathrm{Bcl} 2$.

Conclusion FRP overexpression maintains EC viability by preventing apoptosis via $\mathrm{Bcl} 2$ upregulation. FRP may be a novel therapeutic target for the prevention and treatment of vascular EC injury and of atherosclerosis.

\section{Q0345 SERUM LIPOPROTEIN (A) IS POSITIVELY CORRELATED WITH CORONARY ARTERY CALCIFICATION IN LOW RISK CHINESE PATIENTS}

doi:10.1136/hrt.2010.208967.345

Chengxing Shen, Jun Bao, Xiaoxia Chen, Genshan Ma. Zhongda Hospital southeast University

Background Prior studies indicated that lipoprotein (a) is an independent risk factor for coronary atherosclerosis, but the relationship of serum lipoprotein (a) and coronary artery calcification is still poorly understood in Chinese population.

Objective The present study is to investigate the human lipid profile of a single center (lipoprotein (a), other blood lipid levels) with the relationship of coronary artery calcification.

Method 388 patients suspected with coronary artery disease under coronary CT examinations from March 2007 to June 2009 in our 